\title{
Delivery of Mesenchymal Stem Cells in Chitosan/Collagen Microbeads for Orthopedic Tissue Repair
}

\author{
Limin Wang Rameshwar R. Rao Jan P. Stegemann \\ Department of Biomedical Engineering, University of Michigan, Ann Arbor, Mich., USA
}

\section{Key Words}

Cell therapy · Chitosan · Collagen · Microencapsulation .

Modular tissue engineering

\begin{abstract}
Microencapsulation and delivery of stem cells in biomaterials is a promising approach to repairing damaged tissue in a minimally invasive manner. An appropriate biomaterial niche can protect the embedded cells from the challenging environment in the host tissue, while also directing stem cell differentiation toward the desired lineage. In this study, adult human mesenchymal stem cells (MSC) were embedded in hydrogel microbeads consisting of chitosan and type I collagen using an emulsification process. Glyoxal and $\beta$-glycerophosphate were used as chemical and physical crosslinkers to initiate copolymerization of the matrix materials. The average size and size distribution of the microbeads could be varied by controlling the emulsification conditions. Spheroidal microbeads ranging in diameter from 82 \pm 19 to $290 \pm 78 \mu \mathrm{m}$ were produced. Viability staining showed that MSC survived the encapsulation process ( $>90 \%$ viability) and spread inside the matrix over a period of 9 days in culture. Induced osteogenic differentiation using medium supplements showed that MSC increased gene expression
\end{abstract}

of osterix and osteocalcin over time in culture, and also deposited calcium mineral. Bone sialoprotein and type I collagen gene expression were not affected. Delivery of microbeads through standard needles at practically relevant flow rates did not adversely affect cell viability, and microbeads could also be easily molded into prescribed geometries for delivery. Such protein-based microbeads may have utility in orthopedic tissue regeneration by allowing minimally invasive delivery of progenitor cells in microenvironments that are both protective and instructive.

Copyright $\odot 2013$ S. Karger AG, Basel

\section{KARGER}

E-Mail karger@karger.com

www.karger.com/cto
(C) 2013 S. Karger AG, Basel

$1422-6405 / 13 / 1975-0333 \$ 38.00 / 0$ 


\section{Introduction}

Cell-based therapies for enhancing bone regeneration are being actively pursued, due to the limitations of current approaches in treating recalcitrant and poorly vascularized bone wounds [Rahaman and Mao, 2005; Johnson et al., 2011]. However, a purely cellular approach is not always appropriate for large bone defects, and as a result, there has been increasing interest in using a combination of cells and matrix in these applications [Gloria et al., 2010; Costa-Pinto et al., 2011]. The addition of a defined extracellular matrix provides space-filling properties to the cellular implant and can also serve as a delivery vehicle. The concept of 'modular tissue engineering' has consequently evolved as an approach to creating instructive cellular niches for use in regenerative medicine [Nichol and Khademhosseini, 2009]. These efforts embrace advances in biology and materials science to control the cellular microenvironment in individual modular tissue units, which can be further assembled to form larger tissue structures [Leung and Sefton, 2010; Cheng et al., 2011; Du et al., 2011].

Embedding of stem and progenitor cells in appropriate biomaterials has been used widely to maintain their self-renewal [Zhu et al., 2009] and direct their function [Kaplan et al., 2009; Lund et al., 2009; Marklein and Burdick, 2010; Giobbe et al., 2012]. Control of the composition of the matrix that surrounds the cells creates microenvironments that regulate cell-matrix interactions and can therefore potentially be used to control cell differentiation. Naturally derived polysaccharides and proteins are often used in these approaches because of their biological relevance and biocompatibility. For example, the polysaccharides alginate and agarose have been used for chondrocyte culture and cartilage regeneration because of their chemical similarity to glycosaminoglycans [Kessler and Grande, 2008]. Both of these matrices have demonstrated the ability to prevent chondrocyte dedifferentiation, as well as promoteredifferentiation of dedifferentiated cells [Hauselmann et al., 1992; Barlic et al., 2008]. Alginate microbeads have also been used to culture mesenchymal stem cells (MSC), including bone repair applications [Abbah et al., 2008; Penolazzi et al., 2010]. However, this matrix does not provide adhesive ligands for MSC, and therefore, is not conducive to osteogenic differentiation. Chitosan is an animated polysaccharide that has been used in bone tissue engineering because of its cell adhesive properties, and it has been shown to support osteogenesis of MSC [Peschel et al., 2012].
Extracellular matrix proteins contain bioactive and cell adhesive sequences that play critical roles in controlling cell function. In our previous work, we demonstrated the ability to supplement agarose microbeads with type I collagen using a water-in-oil emulsification process [Batorsky et al., 2005]. In these agarose/collagen microbeads, type I collagen acted as a bioactive component that supported cell attachment and proliferation and served to initiate osteogenic differentiation [Lund et al., 2008]. Human bone marrow-derived MSC embedded in these materials attached to the type I collagen spread inside the matrix and exhibited an upregulation of osteogenic genes and calcium deposition even in the absence of typical osteogenic medium supplements. However, the maximum collagen content that could practically be incorporated in the matrix was about $40 \mathrm{wt} \%$, beyond which microbeads became fragile.

In an effort to produce more robust matrices that support osteogenic differentiation of MSC, we examined chitosan/collagen materials. Our approach was to initiate gelation of both chitosan and collagen using $\beta$-glycerophosphate $(\beta-\mathrm{GP})$ to create composite matrices [Wang and Stegemann, 2010b]. We showed that copolymerization of chitosan and collagen occurred at physiological $\mathrm{pH}$ and a temperature with over $90 \%$ viability of embedded MSC. In contrast to the relatively inert agarose, the presence of chitosan in the matrix stimulated osteogenic differentiation by upregulating the osterix and bone sialoprotein genes. In addition, we used the small dialdehyde glyoxal to crosslink chitosan/collagen matrices in the presence of cells, resulting in a 6-fold increase in mechanical stiffness [Wang and Stegemann, 2011]. The osteogenic properties of chitosan combined with the cell adhesive properties of collagen make these materials attractive for bone tissue engineering applications.

Our intent in the current study was to develop new types of engineered tissue modules based on these chitosan/collagen materials. The emulsification method used to make early microbead formulations was adapted to create chitosan/collagen microbeads containing embedded human bone marrow-derived MSC. The resulting microbeads and the MSC embedded within them were characterized in terms of their morphology, size, viability and osteogenic differentiation. In addition, we examined the use of chitosan/collagen microbeads as vehicles for cell delivery, based on their suitability to be extruded through standard needles and their ability to form cohesive pastes and molded constructs. Such protein-based microbeads may have utility in creating engineered orthopedic tissues and, in particular, could provide a mini- 
mally invasive method for transplanting predifferentiated cells that are contained within a protective and instructive microenvironment.

\section{Materials and Methods}

\section{Microbead Fabrication}

Ultrapure chitosan (93\% degree of deacetylation, molecular weight 267,000, product number CH001; BioSyntech Inc., Quebec, Que., Canada) was dissolved in $0.1 \mathrm{~N}$ acetic acid (Sigma-Aldrich Inc., St. Louis, Mo., USA) at a concentration of $2.0 \mathrm{wt} \%$ and then sterilized by autoclave. Bovine type I collagen (product number 150026; MP Biomedicals, Solon, Ohio, USA,) was dissolved in 0.02 $\mathrm{N}$ acetic acid at $4.0 \mathrm{mg} / \mathrm{ml}$. $\beta$-GP (Sigma-Aldrich) and glyoxal (Sigma-Aldrich) were used as physical and chemical crosslinkers, respectively, to initiate copolymerization of chitosan and collagen, as described previously [Wang and Stegemann, 2011]. The final composition of the matrix mixture included specified chitosan/ collagen ratios (wt/wt: $35 / 65,50 / 50,65 / 35$ ), as well as $5.0 \% \beta$-GP and $0.5 \mathrm{~mm}$ glyoxal. MSC were encapsulated at $1 \times 10^{6} \mathrm{cells} / \mathrm{ml}$. Chitosan/collagen composite microbeads were fabricated using a water-in-oil emulsification process (fig. 1) modified from a previous protocol [Batorsky et al., 2005]. Briefly, a mixture of the desired ratios of chitosan, collagen, $\beta-G P$, glyoxal and MSC was kept on ice prior to being injected into a liquid polydimethylsiloxane (PDMS) emulsification bath precooled to $0^{\circ} \mathrm{C}$ on ice. The bath was stirred with an impeller for 3 min to obtain a homogenous waterin-oil emulsion, after which the temperature was raised to $37^{\circ} \mathrm{C}$ for 25 min to allow simultaneous gelation of the chitosan and collagen matrices. Microbeads formed in the PDMS phase were collected by centrifugation at $200 \mathrm{~g}$ for $5 \mathrm{~min}$ and then washed in phosphate-buffered saline (PBS) three times. Microbeads were immediately assayed after fabrication and were not stored prior to further characterization and cell culture.

\section{Microbead Diameter, Size Distribution and Morphology}

The 50/50 chitosan/collagen composite formulation was used to evaluate microbead diameter, size and size distribution as well as morphology. Impeller speeds were increased from 600 to 1,200 $\mathrm{rpm}$ in increments of $200 \mathrm{rpm}$ to determine the effect on microbead size. Collected microbeads were imaged under a light microscope, and microbead diameter was determined using image analysis software (Image-Pro, Media Cybernetics, Bethesda, Md., USA). Microbeads were also stained with Coomassie blue reagent (Sigma-Aldrich) to visualize the incorporation and distribution of type I collagen in the composite matrix.

\section{Cell Viability}

MSC (product number PT-2501; Lonza Inc., Walkersville, Md., USA) at passage 1 were expanded to passage 5 in complete medium composed of low glucose Dulbecco's Modified Eagle Medium (Invitrogen, Carlsbad, Calif., USA), 10\% MSC-qualified fetal bovine serum (Invitrogen) and 1\% penicillin/streptomycin (Invitrogen). Chitosan/collagen microbeads with specified matrix ratios $(35 / 65$, $50 / 50,65 / 35$ chitosan/collagen) were fabricated and cultured in complete medium for 9 days. Cell viability was visualized using a vital stain (Live/Dead ${ }^{\circledR}$, Invitrogen) at days 1 and 9. For vital staining, microbeads were washed three times in PBS for $10 \mathrm{~min}$ and

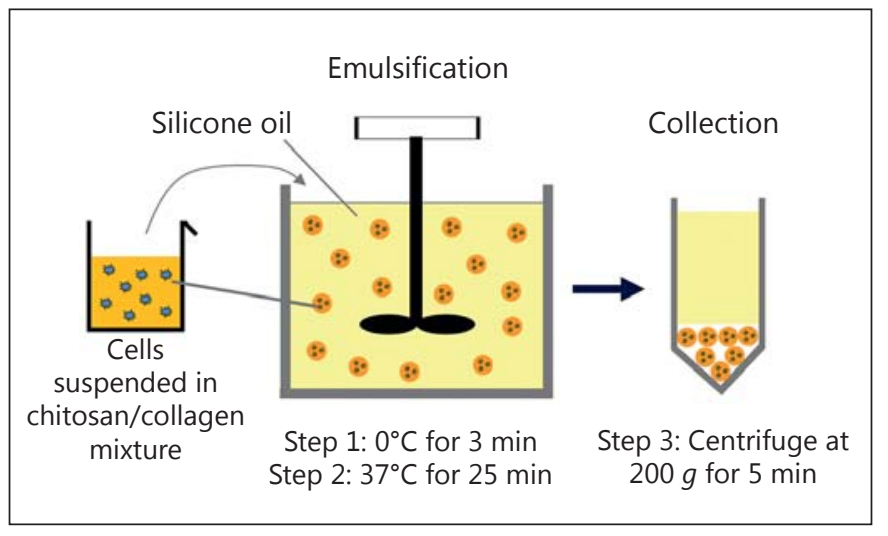

Fig. 1. Schematic of microbead fabrication using water-in-oil emulsification.

were then incubated for $30 \mathrm{~min}$ at $37^{\circ} \mathrm{C}$ with $4 \mu \mathrm{M}$ calcein acetoxymethyl ester and $4 \mu \mathrm{M}$ ethidium homodimer in PBS. The samples were then washed again and imaged on a confocal fluorescent microscope (Olympus FluoView 500 Laser Scanning Confocal Microscope; Olympus America, Center Valley, Pa., USA). Viability was assessed by manually examining multiple representative images from each treatment, and the respective percentage of live cells (cytoplasm stained green) and dead cells (nuclear stained red) was estimated in 5\% increments for each sample type.

To simulate delivery of microbead preparations through a needle and to examine the effect of shear stress during injection on cell viability, microbeads were first concentrated by briefly centrifuging at $200 \mathrm{~g}$ and removing the supernatant medium. Concentrated microbeads with a 50/50 chitosan/collagen ratio were then loaded into syringes and extruded through 0.5 -inch-long needles of three different sizes: 16 gauge (1,194 $\mu \mathrm{m}$ inner diameter), 20 gauge $(584 \mu \mathrm{m}$ inner diameter) and 25 gauge $(241 \mu \mathrm{m}$ inner diameter). Injection rate was controlled by a digital syringe pump, and rates of $0.1 \mathrm{ml} / \mathrm{s}$ (slow injection) and $1.0 \mathrm{ml} / \mathrm{s}$ (rapid injection) were tested. Cell viability was examined before and after injection, as described above.

\section{Osteogenic Differentiation}

Composite microbeads containing the $50 / 50$ chitosan/collagen ratio were fabricated for osteogenic differentiation. Passage 5 MSC were encapsulated in the microbeads at $1 \times 10^{6} \mathrm{cells} / \mathrm{ml}$ and cultured for 15 days in an osteogenic medium composed of the complete medium supplemented with $100 \mathrm{nM}$ dexamethasone (SigmaAldrich), $5 \mu \mathrm{M} \beta$-GP and $50 \mu \mathrm{g} / \mathrm{ml}$ ascorbic acid 2-phosphate (Sigma-Aldrich). Cells were also cultured in the complete medium without osteogenic supplements, serving as a control group. At days 1 and 15, samples were snap-frozen in liquid nitrogen and stored at $-80^{\circ} \mathrm{C}$. All frozen samples were pulverized in a BioPulverizer (Biospec Inc., Bartlesville, Okla., USA) for the following assays.

\section{RNA Isolation and Gene Expression Assays}

A cetyltrimethylammonium bromide-based method was used to isolate RNA from the pulverized samples $(n=4)$. This method has been shown to be superior to conventional methods in yielding 

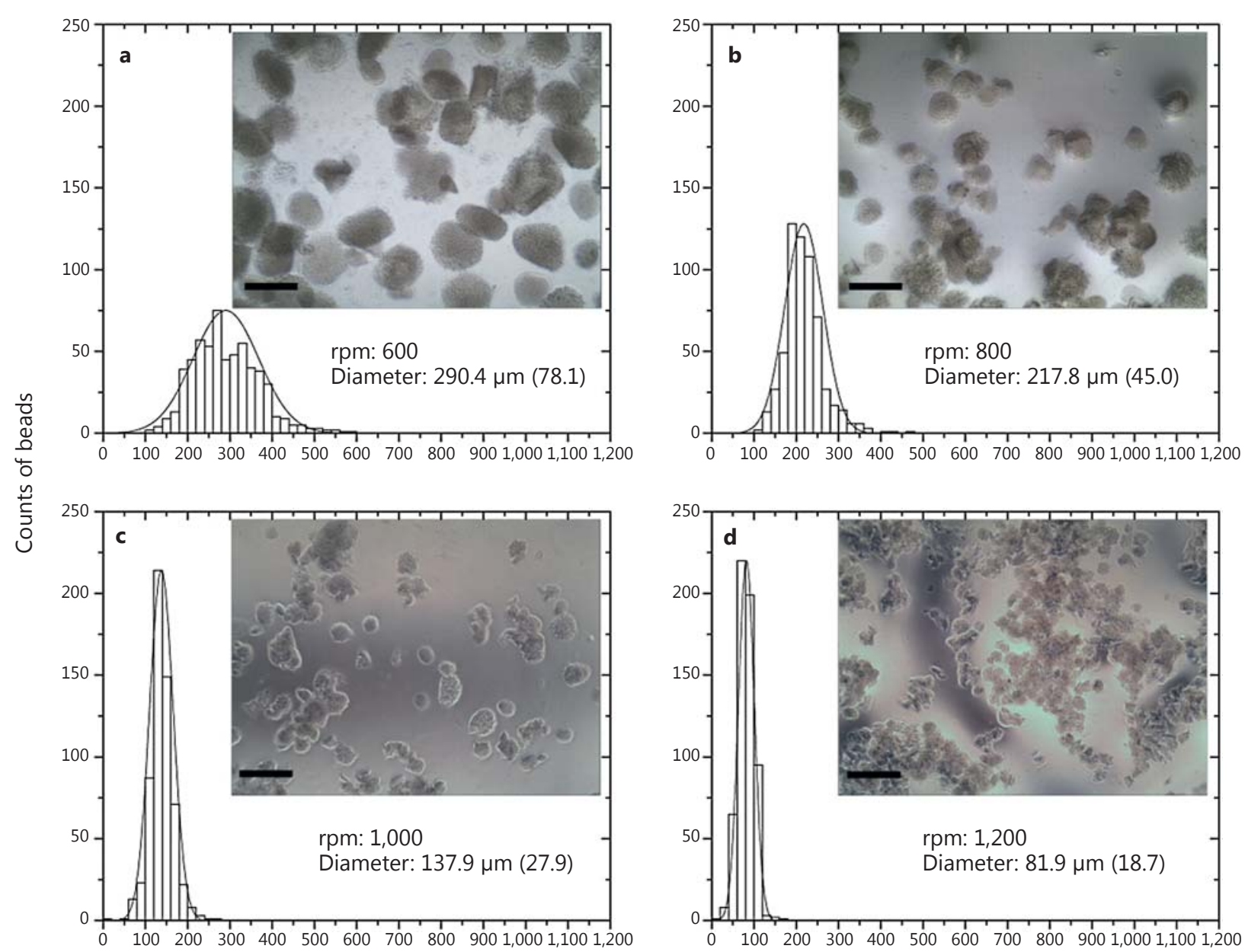

Diameter $(\mu \mathrm{m})$

Fig. 2. Average size and size distribution of chitosan/collagen microbeads created using varying emulsification impeller speeds (rpm). Average diameters are shown, with standard deviation in parentheses. Insets show light microscopy images of each microbead preparation. Scale bar $=300 \mu \mathrm{m}$.

high-quality RNA from polysaccharide matrices, as described previously [Wang and Stegemann, 2010a]. Briefly, $600 \mu \mathrm{l}$ of prewarmed cetyltrimethylammonium bromide extraction buffer was mixed with pulverized sample powders and then extracted with an equal volume of chloroform-isoamyl alcohol (24:1; Fisher Scientific, Pittsburgh, Pa., USA). Total RNA was precipitated with an equal amount of isopropanol (Sigma-Aldrich), washed in $75 \%$ ethanol and dissolved in $30 \mu \mathrm{l}$ RNase-free water. Subsequently, a Qiagen RNeasy Mini kit (Qiagen Inc., Valencia, Calif., USA) was used to further purify the obtained RNA.

For quantitative reverse transcriptase-polymerase chain reaction, cDNA was obtained using a High-Capacity cDNA Archive kit (Applied Biosystems Inc., Foster City, Calif., USA) following the manufacturer's instruction. TaqMan gene expression assay kits (Applied Biosystems) were used to assay for glyceraldehyde 3-phosphate dehydrogenase (GAPDH, Hs99999905_m1), type I collagen (CI, Hs00164004_m1), bone sialoprotein (BSP, Hs00173720_m1), osteocalcin (OCN, Hs01587813_g1) and osterix (OSX, Hs00541729_m1) in an Applied Biosystems 7500 Fast System. Gene expression data were first normalized to Ct values of the GAPDH gene and then normalized again to their respective $\mathrm{Ct}$ values at day 1 .

\section{Calcium Quantification}

Calcium content was detected by an OCPC (orthocresolphthalein complexone) method as previously described [Wang et al., 2010]. Pulverized sample powders $(n=4)$ were dissolved in $1.0 \mathrm{~N}$ acetic acid overnight. Twenty microliters of the digested solution 

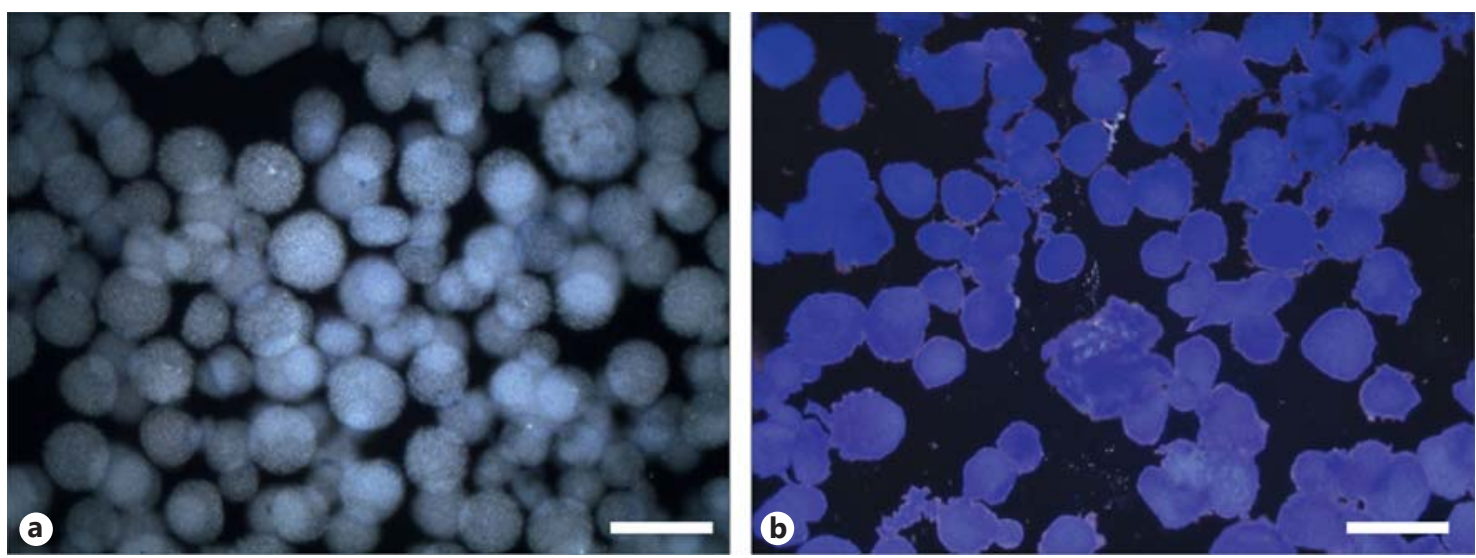

Fig. 3. Coomassie blue protein staining in pure chitosan (a) and chitosan/collagen $(\mathbf{b})$ microbeads. Scale bar $=$ $300 \mu \mathrm{m}$.

was incubated with $250 \mu \mathrm{l}$ of working solution consisting of 0.05 $\mathrm{mg} / \mathrm{ml}$ OCPC solution and ethanolamine/boric acid/8-hydroxyquinoline buffer (Sigma-Aldrich) for $10 \mathrm{~min}$ at room temperature. The plate was then read spectrophotometrically at $405 \mathrm{~nm}$. Calcium content was normalized to DNA content measured by the PicoGreen ${ }^{\circledR}$ kit (Invitrogen) following the manufacturer's protocol.

\section{Statistical Analysis}

Quantitative data were expressed as the mean \pm standard deviation and analyzed by analysis of variance and Tukey's post hoc tests. Statistical differences were determined by a level of significance of $\mathrm{p}<0.05$.

\section{Results}

\section{Microbead Size and Morphology}

The emulsification process produced irregular though generally spherical microbeads, as shown in figure 2 . Microbead size varied inversely with the impeller speeds used for emulsification (fig. 2). Specifically, average microbead size decreased from about $290 \mu \mathrm{m}$ at $600 \mathrm{rpm}$ to around $80 \mu \mathrm{m}$ at 1,200 rpm. Microbeads were well dispersed in the PDMS phase prior to collection; however, they tended to aggregate in aqueous solution after collection. Aggregation was more evident in the smaller microbeads, particularly the group with the smallest diameter (made at 1,200 rpm; fig. 2d). The size distribution of microbead populations became narrower with increasing the impeller speeds, as reflected by the decrease in the standard deviation of the microbead size range. The ratio of chitosan/collagen did not appreciably affect microbead size over the range tested. Figure 3 shows staining of the protein (collagen) component of the microbeads using
Coomassie blue stain. Pure chitosan microbeads did not stain (fig. 3a), while the intense blue staining in 50/50 chitosan/collagen microbeads revealed the type I collagen protein matrix (fig. 3b). The uniform blue staining of the chitosan/collagen microbeads suggested a homogenous distribution of type I collagen.

\section{Cell Viability and Morphology}

Figure 4 shows viability staining of MSC inside chitosan/collagen microbeads at days 1 and 9 in culture. The abundance of green staining (and lack of red-stained nuclei) in all groups at day 1 indicated that MSC survived the fabrication process with high cell viability (estimated at $>90 \%$ ). Cell spreading was observed in microbeads with chitosan/collagen ratios of 35/65 and 50/50, even at day 1 , though cells in the 65/35 formulation remained rounded. At day 9, high cell viability was maintained in the groups with chitosan/collagen ratios of 35/65 and $50 / 50$, and the cells inside the microbeads were clearly spread and interacting with the microbead matrix. In contrast, cells in $65 / 35$ chitosan/collagen microbeads remained rounded and viability was lower, as revealed by red-stained nuclei of dead cells.

\section{Osteogenic Differentiation in Microbeads}

Figure 5 shows gene expression and calcium deposition of MSC embedded in 50/50 chitosan/collagen microbeads. A set of osteogenic genes (BSP, CI, OCN, OSX) were examined at days 1 and 15, in the presence or absence of osteogenic medium supplements (fig. 5a). Over 15 days, OCN expression increased significantly ( $\mathrm{p}<$ 0.05 , approximately 3 -fold increase relative to day 1 ) in 


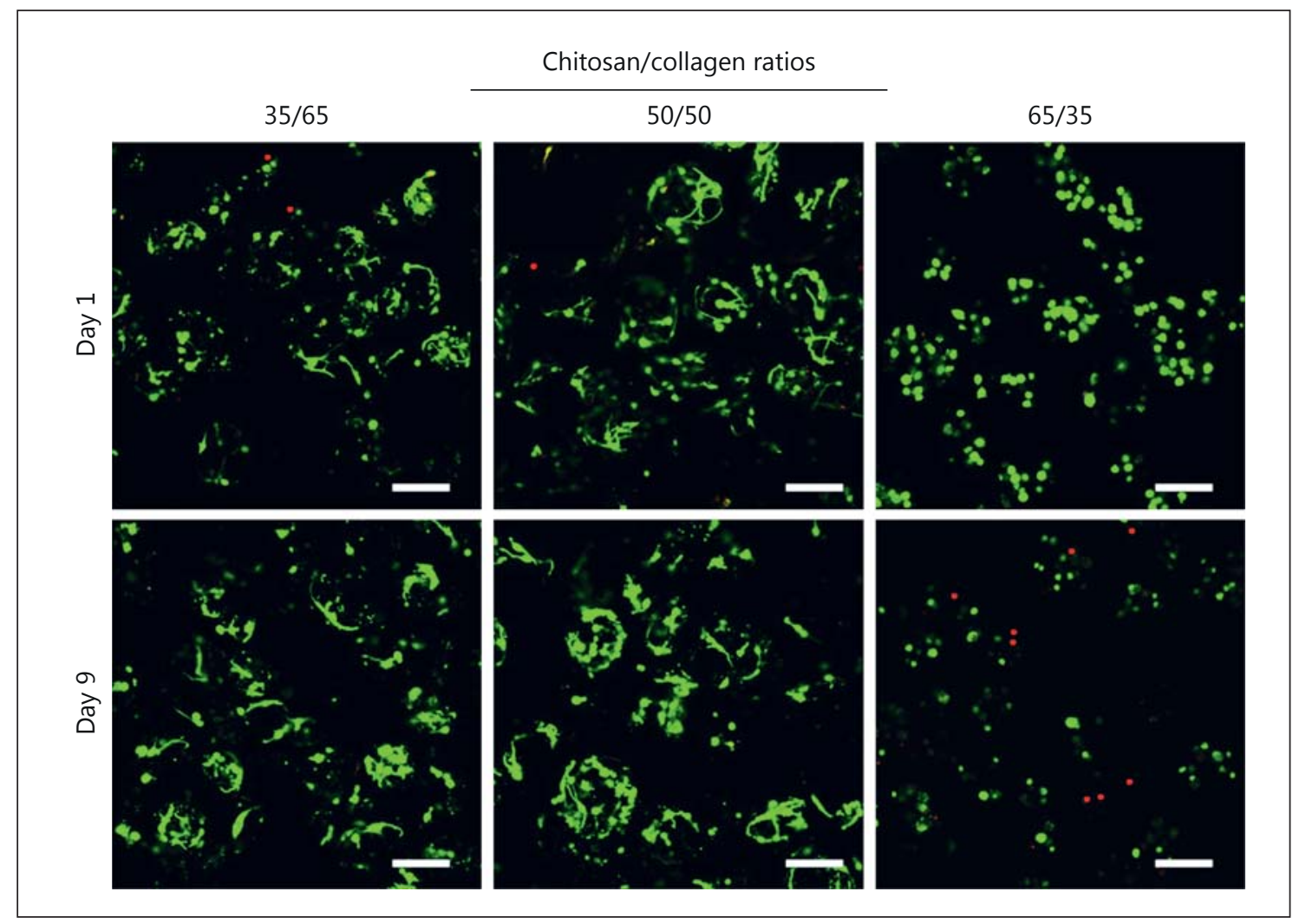

Fig. 4. Viability staining of MSC embedded in microbeads with varying chitosan/collagen ratios at days 1 and 9 in culture. Scale bar $=200 \mu \mathrm{m}$. The cytoplasm of living cells is stained green and the nucleus of dead cells is stained red.

the osteogenic group, whereas the control group did not show a statistically significant change. OSX gene expression in both osteogenic and control groups increased significantly with time $(\mathrm{p}<0.05)$, exhibiting about a 5 -fold increase in control medium and a 13 -fold increase in osteogenic medium. At day 15, both OCN and OSX gene levels were significantly higher in the osteogenic group, relative to the control group ( $\mathrm{p}<0.05)$. BSP and CI gene expression in both the osteogenic and control groups showed no statistically significant change over 15 days, nor was there a difference between the osteogenic and control groups in these genes.

Calcium deposition was quantitatively measured to evaluate the initial stages of mineralization (fig. 5b). The osteogenic group exhibited a significant increase in calcium content from days 1-15 and also produced markedly more calcium (approximately 300 -fold increase on a per cell basis) than the control group at day 15 ( $\mathrm{p}<0.05)$. The calcium content in the control group remained low throughout the 15-day culture period.

\section{Injectability and Moldability}

Figure 6 shows cell viability of MSC embedded in 50/50 chitosan/collagen microbeads after concentration into a paste and injection through needles of various gauges. These data are for the high injection rate (1.0 $\mathrm{ml} / \mathrm{s}$ ) and show that cell viability remained high and was not significantly affected by injection through any of the needles tested. Data for the lower injection rate (0.1 $\mathrm{ml} / \mathrm{s}$ ) were similar (data not shown). Figure 7 shows images of 50/50 chitosan/collagen microbead preparations being molded and handled for delivery. Centrifugation was used to concentrate the microbeads, and depending on the amount of medium that was removed, the pastes could be extruded as well-hydrated slurries (fig. 7a) or as more concentrated pastes (fig. $7 \mathrm{~b}$ ) that demonstrated a very strong degree of cohesion when extruded through a needle (fig. 7c). Microbead pastes could also be molded into desired shapes, either through direct centrifugation into a mold (fig. 7d) or by sculpting of desired geometric shapes (fig. 7e). Molded chitosan/collagen mi- 


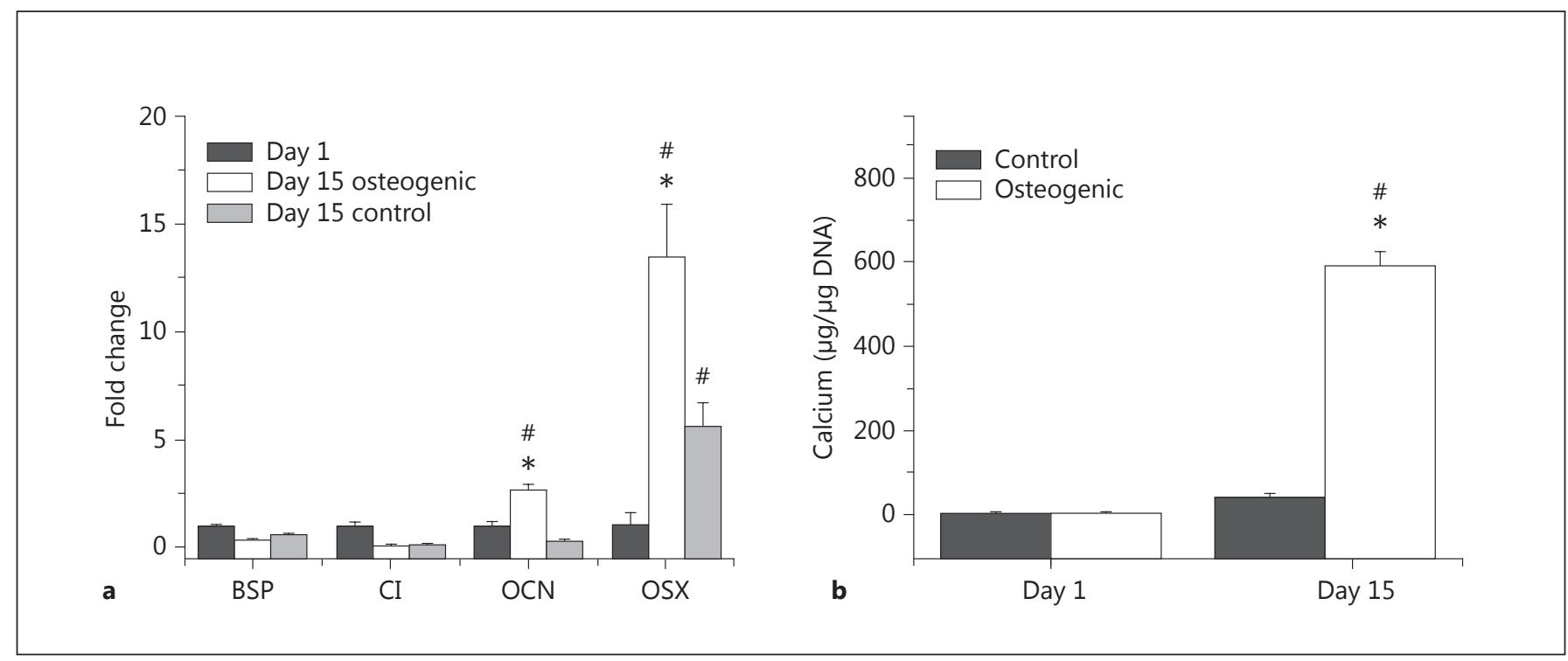

Fig. 5. Gene expression (a) and calcium deposition (b) during osteogenic differentiation of MSC in chitosan/collagen microbeads. * Statistically significant difference from control group; ${ }^{*}$ statistically significant difference from day 1.

crobead pastes also showed strong cohesiveness and could be easily handled with surgical instruments (fig. 7f).

\section{Discussion}

The method we have developed to create composite chitosan/collagen microbeads for cell encapsulation and delivery is flexible and facile. It is a batch emulsification process that produces an entire population of microbeads simultaneously, and microbead preparations from 1 to 25 $\mathrm{ml}$ can easily be made using a bench-scale apparatus. The size of the individual microbeads and the size distribution of the microbead population can be controlled by varying the impeller speed. In this study, average bead diameter was varied from approximately 80 to $300 \mu \mathrm{m}$, using impeller speeds from 600 to $1,200 \mathrm{rpm}$, and the size distribution became narrower with increasing impeller speed. The size of the microbeads is important for several reasons. By controlling the number of cells to be encapsulated and the resulting microbead size, the number of cells per microbead can be defined. When in a rounded morphology, the diameter of MSC is around $20 \mu \mathrm{m}$; however, these cells typically spread in culture to reach dimensions of $80-100 \mu \mathrm{m}$. Therefore, we aimed to create microbeads
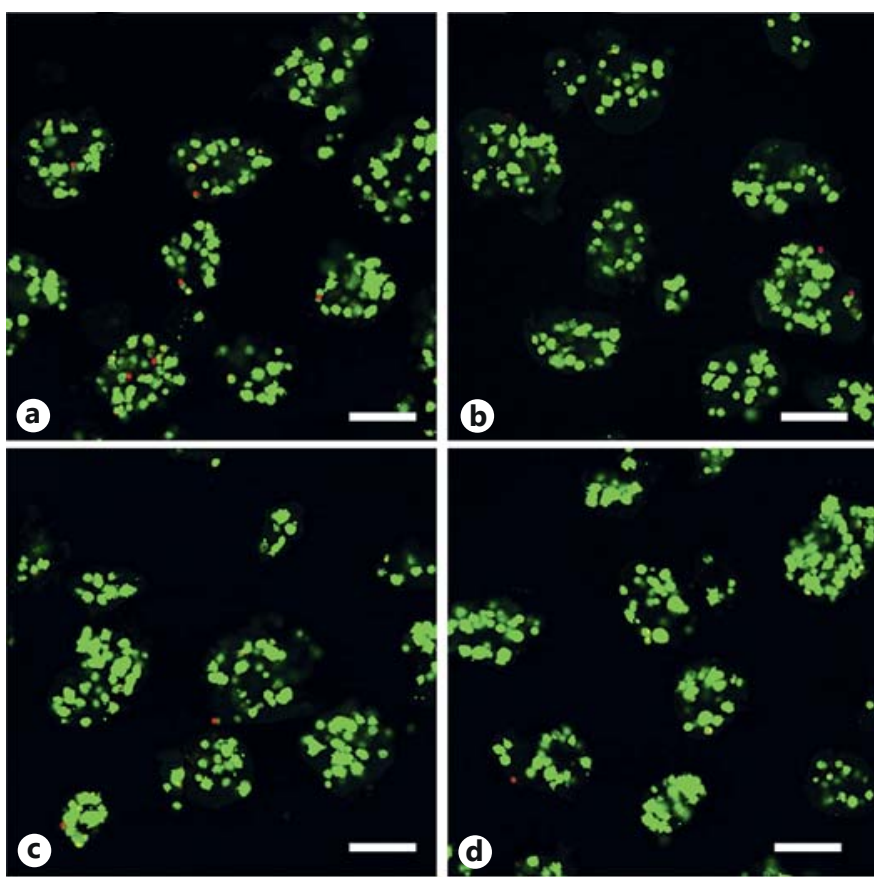

Fig. 6. Viability of MSC embedded in chitosan/collagen microbeads before injection (a) and after passing through 16- (b), 20- (c) and 25 -gauge $(\mathbf{d})$ needles (length $0.5 \mathrm{inch}$ ) at a flow rate of $1.0 \mathrm{ml} / \mathrm{s}$. Scale bar $=200 \mu \mathrm{m}$. 

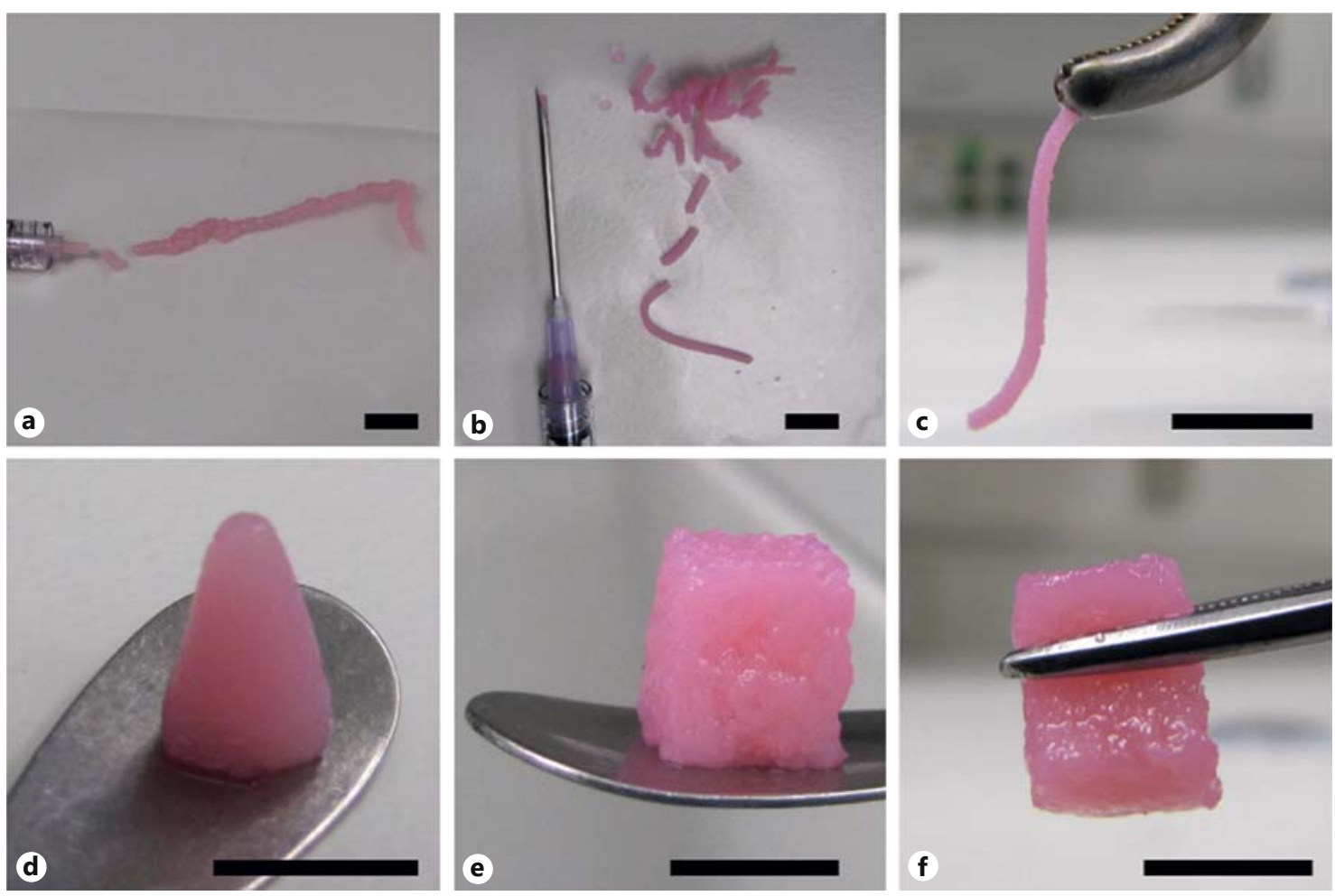

Fig. 7. Images of chitosan/collagen microbead pastes after injection from a syringe (a) or standard needle (b). Extruded pastes are highly cohesive (c) and can be molded into desired geometries by centrifugation (d) or sculpting (e). Microbead constructs can be handled with surgical tools $(\mathbf{c}, \mathbf{f})$. Scale bar in each panel $=1 \mathrm{~cm}$.

that would encapsulate MSC and also provide sufficient space for cell spreading, while minimizing the diffusion length between the cells and the microbead surface. Microbead size and size distribution are also important because they can be used to control the packing density of concentrated bead preparations. Very uniform bead populations will have relatively large void spaces between beads, whereas microbeads with a wider size distribution will pack together more closely to create higher density pastes. The overall density of a microbead paste will affect mass transfer and possibly neovascularization of the material when implanted.

Maintenance of high cell viability during and following the encapsulation process is critical to ensure that the embedded cells can engraft and function at the repair site. Therefore, we designed the microbead fabrication method to avoid materials and conditions detrimental to isolated cells, and indeed, vital staining showed that MSC were not harmed by the fabrication process. PDMS has been used in a variety of cell-contacting applications [Yeh et al., 2006; Kilian et al., 2010] and has been shown to be cytocompatible. The level of fluid shear induced by the emulsification impeller, though difficult to quantify, did not damage cells. In addition, the temperature range used in our process was from approximately 4 to $37^{\circ} \mathrm{C}$, which cells can tolerate, and the $\mathrm{pH}$ was maintained at close to physiological levels. The choice of the chitosan/collagen matrix concentration and ratio was influenced by our previous studies using these materials in bulk gel format [Wang and Stegemann, 2010b]. In the present study, the $50 / 50$ chitosan/collagen formulation was of particular interest because it allowed cell spreading while producing robust microbeads.

The use of $\beta$-GP to initiate gelation in the emulsification bath allowed us to create a stable chitosan/collagen matrix surrounding the cells before collection of the microbeads. Although the mechanism is not fully understood, gelation can be attributed to $\mathrm{pH}$-dependent changes in the ionic strength between $\beta$-GP and chitosan and proton transfer between these materials [Chenite et al., 2000, 2001; Cho et al., 2005]. The microbead matrix was further stabilized through crosslinking with low concen- 
trations of glyoxal, a small aldehyde that we have used in previous studies, and which has been shown to be cytocompatible at low concentrations [Wang and Stegemann, 2011]. Overall, the combination of physical and chemical parameters used to fabricate chitosan/collagen microbeads was shown to support cell survival, while also producing mechanically robust engineered tissue modules.

After fabrication, the composition and properties of the matrix surrounding the cells can impact both their survival and function. In this study, MSC embedded in $35 / 65$ and 50/50 chitosan/collagen composite microbeads clearly interacted with and spread inside the 3D matrix and maintained high cell viability over 9 days. In contrast, MSC embedded in 65/35 microbeads retained a rounded morphology, and a marked decrease in cell viability was evident by day 9 in culture. Chitosan and type I collagen have both been used in a variety of cell therapy approaches; however, they have very different interactions with cells. Collagen is a protein with well-characterized binding sites for cells, and most mammalian cells can produce and remodel collagen. In contrast, chitosan is a polysaccharide that does not present cell-binding sites. Therefore, microbeads with higher collagen content are more conducive to spreading of cells, which has been linked to osteogenic differentiation in MSC [McBeath et al., 2004]. Microbeads with a higher chitosan content did not exhibit sufficient cell-matrix interactions to promote spreading, which may also lead to eventual cell death because of a lack of environmental cues. However, our previous work has shown that chitosan can present an osteogenic stimulus to MSC [Wang and Stegemann, 2011] and also serves to stabilize the microbeads. Therefore, our intent in this study was to combine chitosan and collagen to capitalize on favorable properties of each material.

Osteogenic differentiation of MSC is accompanied by the upregulation of osteogenic gene expression and mineral deposition. In the present study, we examined four genes that are markers of the osteogenic lineage. The transcription factor OSX is highly specific to osteoblastic differentiation and bone formation, both in development and postnatally [Nakashima et al., 2002; Zhou et al., 2010]. MSC in chitosan/collagen microbeads cultured in osteogenic medium expressed markedly higher OSX gene levels at day 15 , relative to day 1 , showing that these materials support osteogenic differentiation. Interestingly, microbeads in control medium also exhibited elevated OSX gene expression at day 15, suggesting that chitosan/ collagen composite materials themselves may be osteoinductive. The extracellular matrix protein osteocalcin is mainly secreted by mature osteoblasts at the late stage of

Delivery of MSC in Chitosan/Collagen Microbeads osteoblastic differentiation, and its expression has been shown to coincide with mineralization [Lian et al., 1998]. In our study, we observed both upregulation of OCN expression and increased calcium deposition in osteogenic medium at day 15 . In contrast, in control medium, we saw no increase in either $\mathrm{OCN}$ or calcium deposition. These results highlight the importance of ascorbic acid and $\beta$-GP in the osteogenic medium, since these components are required for expression of the OCN gene and the mineralization by osteoblasts. The extracellular matrix proteins bone sialoprotein and type I collagen are both tightly associated with hydroxyapatite nucleation during the mineralization process [Hunter and Goldberg, 1994]. Expression of these genes was not significantly affected by culture in chitosan/collagen microbeads in the present study. In previous studies, we have observed that BSP is typically upregulated at later time points than examined in this work, and that CI gene expression tends to be inhibited by culture in matrices that contain exogenously added type I collagen [Lund et al., 2008].

A main advantage of the microbead format is that it provides a biomaterial-based approach to delivering cells in a tailored microenvironment. In this respect, one function of the chitosan/collagen matrix that surrounds the cells is to protect them during and after delivery. In the current study, extrusion of microbead pastes from standard syringes and needles did not adversely affect the viability of cells in microbeads. It has been shown that direct injection of naked cells can reduce viability and that hydrogel carriers can prevent this effect [Aguado et al., 2012]. This protective effect may continue in vivo after microbead injection or implantation, since the defined matrix may promote cell survival and desired differentiated functions. Therefore, microbead embedding of MSC in natural, osteoconductive materials offers a practical approach to efficiently delivering viable cells in a minimally invasive manner to diseased or damaged bone tissues. The modular microbead format also has advantages in terms of facilitating mass transfer through the void spaces between beads, which may also serve as areas for host cell and capillary invasion. This type of injectable therapy may be particularly useful in challenging bone regeneration applications such as avascular necrosis, implant fixation and percutaneous vertebroplasty.

The field of modular tissue engineering is growing, as new biomaterials and processing methods are developed and used to create microscale tissue constructs. This approach has recently been applied to a variety of tissues, including bone [Dormer et al., 2012; Chatterjea et al., 2013], cartilage [Cheng et al., 2011] and vasculature 
[Chamberlain et al., 2012; Chen et al., 2013]. In the area of bone tissue engineering, a number of materials have been used in the modular format, including alginate [Grellier et al., 2009; Man et al., 2012], calcium phosphate [Jin et al., 2012] and composites [Zhou et al., 2011]. Such modular microconstructs offer the very attractive possibility of minimally invasive delivery of living tissue to sites of injury. In addition, the ability to create complex but well-defined macroscale architectures based on microscale subunits is a promising approach to creating multiphase tissues and tissue interfaces [Kachouie et al., 2010; Caldwell et al., 2013]. The chitosan/collagen micro- beads described in the present study have potential in these emerging cell-based approaches to bone regeneration.

\section{Acknowledgements}

This project was supported in part by the National Institute of Arthritis and Musculoskeletal and Skin Diseases through grant R01-AR053231, by a grant from the AO Foundation Large Bone Defect Healing Consortium, and by a National Science Foundation Graduate Research Fellowship (to R.R.R.).

\section{References}

- Abbah SA, Lu WW, Chan D, Cheung KM, Liu WG, et al. (2008) Osteogenic behavior of alginate encapsulated bone marrow stromal cells: an in vitro study. J Mater Sci Mater Med 19: 2113-2119.

-Aguado BA, Mulyasasmita W, Su J, Lampe KJ, Heilshorn SC (2012) Improving viability of stem cells during syringe needle flow through the design of hydrogel cell carriers. Tissue Eng Part A 18: 806-815.

-Baht GS, Hunter GK, Goldberg HA (2008) Bone sialoprotein-collagen interaction promotes hydroxyapatite nucleation. Matrix Biol 27: 600-608.

- Barlic A, Drobnic M, Malicev E, Kregar-Velikonja N (2008) Quantitative analysis of gene expression in human articular chondrocytes assigned for autologous implantation. J Orthop Res 26: 847-853.

Batorsky A, Liao J, Lund AW, Plopper GE, Stegemann JP (2005) Encapsulation of adult human mesenchymal stem cells within collagenagarose microenvironments. Biotechnol Bioeng 92: 492-500.

Caldwell DJ, Rao RR, Stegemann JP (2012) Assembly of discrete collagen-chitosan microenvironments into multiphase tissue constructs. Adv Healthcare Mater, Epub ahead of print.

Chamberlain MD, Gupta R, Sefton MV (2012) Bone marrow-derived mesenchymal stromal cells enhance chimeric vessel development driven by endothelial cell-coated microtissues. Tissue Eng Part A 18: 285-294.

- Chatterjea A, Yuan H, Chatterjea S, Garritsen H, Renard A, van Blitterswijk CA, de Boer J (2013) Engineering new bone via a minimally invasive route using human bone marrowderived stromal cell aggregates, microceramic particles, and human platelet-rich plasma gel. Tissue Eng Part A 3-4: 340-349.

-Chen DY, Wei HJ, Lin KJ, Huang CC, Wang CC, Wu CT, Chao KT, Chen KJ, Chang Y, Sung HW (2013) Three-dimensional cell aggregates composed of HUVECs and cbMSCs for therapeutic neovascularization in a mouse model of hindlimb ischemia. Biomaterials 34 : 1995-2004.

Cheng HW, Luk KD, Cheung KM, Chan BP (2011) In vitro generation of an osteochondral interface from mesenchymal stem cellcollagen microspheres. Biomaterials 32: 1526-1535.

Chenite A, Buschmann M, Wang D, Chaput C, Kandani N (2001) Rheological characterization of thermogelling chitosan/glycerolphosphate solutions. Carbohydr Polym 46: 39-47.

Chenite A, Chaput C, Wang D, Combes C, Buschmann MD, Hoemann CD, Leroux JC, Atkinson BL, Binette F, Selmani A (2000) Novel injectable neutral solutions of chitosan form biodegradable gels in situ. Biomaterials 21: 2155-2161.

-Cho J, Heuzey MC, Begin A, Carreau PJ (2005) Physical gelation of chitosan in the presence of beta-glycerophosphate: the effect of temperature. Biomacromolecules 6: 3267-3275.

- Costa-Pinto AR, Reis RL, Neves NM (2011) Scaffolds based bone tissue engineering: the role of chitosan. Tissue Eng Part B Rev 17: 331347.

Dormer NH, Qiu Y, Lydick AM, Allen ND, Mohan N, Berkland CJ, Detamore MS (2012) Osteogenic differentiation of human bone marrow stromal cells in hydroxyapatite-loaded microsphere-based scaffolds. Tissue Eng Part A 18: 757-767.

Du Y, Ghodousi M, Qi H, Haas N, Xiao W, Khademhosseini A (2011) Sequential assembly of cell-laden hydrogel constructs to engineer vascular-like microchannels. Biotechnol Bioeng 108: 1693-1703.

Giobbe GG, Zagallo M, Riello M, Serena E, Masi G, Barzon L, di Camillo B, Elvassore N (2012) Confined 3D microenvironment regulates early differentiation in human pluripotent stem cells. Biotechnol Bioeng 109: 3119-3132.

Gloria A, De Santis R, Ambrosio L (2010) Polymer-based composite scaffolds for tissue en- gineering. J Appl Biomater Biomech 8: 5767.

Grellier M, Granja PL, Fricain JC, Bidarra SJ, Renard M, Bareille R, Bourget $C$, Amédée J, Barbosa MA (2009) The effect of the co-immobilization of human osteoprogenitors and endothelial cells within alginate microspheres on mineralization in a bone defect. Biomaterials 30: 3271-3278.

Hauselmann HJ, Aydelotte MB, Schumacher BL, Kuettner KE, Gitelis SH, et al. (1992) Synthesis and turnover of proteoglycans by human and bovine adult articular chondrocytes cultured in alginate beads. Matrix 12: 116-129.

-Hunter GK, Goldberg HA (1994) Modulation of crystal formation by bone phosphoproteins: role of glutamic acid-rich sequences in the nucleation of hydroxyapatite by bone sialoprotein. Biochem J 302: 175-179.

Jin GZ, Kim JH, Park JH, Choi SJ, Kim HW, Wall I (2012) Performance of evacuated calcium phosphate microcarriers loaded with mesenchymal stem cells within a rat calvarium defect. J Mater Sci Mater Med 23: 1739 1748.

-Johnson EO, Troupis T, Soucacos PN (2011) Tissue-engineered vascularized bone grafts: basic science and clinical relevance to trauma and reconstructive microsurgery. Microsurgery 31: 176-182.

-Kachouie NN, Du Y, Bae H, Khabiry M, Ahari AF, Zamanian B, Fukuda J, Khademhosseini A (2010) Directed assembly of cell-laden hydrogels for engineering functional tissues. Organogenesis 6: 234-244.

Kessler MW, Grande DA (2008) Tissue engineering and cartilage. Organogenesis 4: 28-32.

-Kilian KA, Bungarija B, Lahn BT, Mrksich M (2010) Geometric cues for directing the differentiation of mesenchymal stem cells. Proc Natl Acad Sci USA 107: 4872-4877.

Leung BM, Sefton MV (2010) A modular approach to cardiac tissue engineering. Tissue Eng Part A 16: 3207-3218. 
Lian JB, Stein GS, Stein JL, van Wijnen AJ (1998) Osteocalcin gene promoter: unlocking the secrets for regulation of osteoblast growth and differentiation. J Cell Biochem Suppl 30-31: $62-72$.

Lund AW, Bush JA, Plopper GE, Stegemann JP (2008) Osteogenic differentiation of mesenchymal stem cells in defined protein beads. J Biomed Mater Res B 87: 213-221.

Lund AW, Yener B, Stegemann JP, Plopper GE (2009) The natural and engineered 3D microenvironment as a regulatory cue during stem cell fate determination. Tissue Eng Part B Rev 15: 371-380.

Man Y, Wang P, Guo Y, Xiang L, Yang Y, Qu Y, Gong P, Deng L (2012) Angiogenic and osteogenic potential of platelet-rich plasma and adipose-derived stem cell laden alginate microspheres. Biomaterials 33: 8802-8811.

Marklein RA, Burdick JA (2010) Controlling stem cell fate with material design. Adv Mater 22: 175-189.

McBeath R, Pirone DM, Nelson CM, Bhadriraju K, Chen CS (2004) Cell shape, cytoskeletal tension, and RhoA regulate stem cell lineage commitment. Dev Cell 6: 483-495.

Nakashima K, Zhou X, Kunkel G, Zhang Z, Deng JM, et al. (2002) The novel zinc finger-containing transcription factor osterix is required for osteoblast differentiation and bone formation. Cell 108: 17-29.
Nichol JW, Khademhosseini A (2009) Modular tissue engineering: engineering biological tissues from the bottom up. Soft Mater 5: 13121319.

-Penolazzi L, Tavanti E, Vecchiatini R, Lambertini E, Vesce F, et al. (2010) Encapsulation of mesenchymal stem cells from Wharton's jelly in alginate microbeads. Tissue Eng Part C Methods 16: 141-155.

Peschel D, Zhang K, Fischer S, Groth T (2012) Modulation of osteogenic activity of BMP-2 by cellulose and chitosan derivatives. Acta Biomater 8: 183-193.

-Rahaman MN, Mao JJ (2005) Stem cell-based composite tissue constructs for regenerative medicine. Biotechnol Bioeng 91: 261-284.

Sundelacruz S, Kaplan DL (2009) Stem cell- and scaffold-based tissue engineering approaches to osteochondral regenerative medicine. Semin Cell Dev Biol 20: 646-655.

-Wang L, Dormer NH, Bonewald LF, Detamore MS (2010) Osteogenic differentiation of human umbilical cord mesenchymal stromal cells in polyglycolic acid scaffolds. Tissue Eng Part A 16: 1937-1948.

Wang L, Stegemann JP (2010a) Extraction of high quality RNA from polysaccharide matrices using cetyltrimethylammonium bromide. Biomaterials 31: 1612-1618.
Wang L, Stegemann JP (2010b) Thermogelling chitosan and collagen composite hydrogels initiated with beta-glycerophosphate for bone tissue engineering. Biomaterials 31: 39763985.

Wang L, Stegemann JP (2011) Glyoxal crosslinking of cell-seeded chitosan/collagen hydrogels for bone regeneration. Acta Biomater 7: 2410-2417.

-Yeh J, Ling Y, Karp JM, Gantz J, Chandawarkar A, Eng G, Blumling J, Langer R, Khademhosseini A (2006) Micromolding of shape-controlled, harvestable cell-laden hydrogels. Biomaterials 27: 5391-5398.

Zhou H, Xu HH (2011) The fast release of stem cells from alginate-fibrin microbeads in injectable scaffolds for bone tissue engineering. Biomaterials 32: 7503-7513.

Zhou X, Zhang Z, Feng JQ, Dusevich VM, Sinha K, Zhang H, Darnay BG, de Crombrugghe B (2010) Multiple functions of Osterix are required for bone growth and homeostasis in postnatal mice. Proc Natl Acad Sci USA 107: 12919-12924.

Zhu Y, Lui T, Song K, Jiang B, Ma X, Cui Z (2009) Collagen-chitosan polymer as a scaffold for the proliferation of human adipose tissue-derived stem cells. J Mater Sci Mater Med 20: 799-808. 